\title{
A Smart Factory Approach to Mass Producing Customised Healthcare Devices for Patients
}

\author{
Urvashi GUNPUTH ${ }^{\mathrm{a}, 1}$, Adam LEIGHTON ${ }^{\mathrm{a}}$, Gavin WILLIAMS ${ }^{\mathrm{a}}$, Paul WOOD ${ }^{\mathrm{a}}$, and \\ Steven ATFIELD ${ }^{\mathrm{b}}$ \\ ${ }^{a}$ Institute for Innovation in Sustainable Engineering, University of Derby, Kedleston \\ Road, Derby, DE22 1GB, UK \\ ${ }^{\mathrm{b}}$ Specialist Rehabilitation Therapies and Specialist Rehabilitation Business Unit \\ Derby and Burton Hospitals Trust, Uttoxeter Road \\ Derby, \\ DE22 3NE, UK
}

\begin{abstract}
Orthoses are devices worn by patients to support their body and are a nonsurgical method for correcting foot and ankle problems. In a national survey, Nester et al, reported in 2017, there are approximately 9 million orthosis users in the UK, and of these 78,000 patients each year need a customised orthosis [1]. The same survey identifies the number of orthoses users in the UK had grown from 1.2 million in 2007, which is nearly an eightfold increase over one decade. The traditional method of fabricating orthoses is extensively craft orientated. The assessment, fabrication, and fitting of an orthosis to a patient is a lengthy process at up to twelve weeks duration and can involve significant engagement with the patient. 3D Printing combined with digital design, laser scanning and gait analysis technologies could dramatically reduce the time of the orthosis fabrication process, accelerate patient healing and reduce cost.
\end{abstract}

Keywords. Smart factory, Additive Manufacture, Orthotic

\section{Introduction}

Ankle foot orthoses are devices worn by patients with long term conditions and/or disabilities aiming to enhance their quality of life without surgical interventions. Receiving the appropriate orthosis in a timely manner is imperative especially for paediatric patients who could outgrow initial measurements taken if there are delays [1]. Such delay could also lead to complications in patients waiting for their orthoses.

Traditional methods of fabricating orthoses in the NHS are extensively craft orientated and performed by specialist technicians. A plaster cast tool of the lower limb is used for thermoforming the main structural element of the orthosis, followed by trimming to a final shape, cutting of cushioning inserts, and assembly the component parts including off-the-shelf components as necessary. The process requires technical skill and significant time and effort before fitting to the patient. It is not uncommon for further refinements to be required following initial patient fitting, typically resulting in extended lead times and further fitting appointments. It can take up to 12 weeks for someone to receive their orthosis $[2,3]$ as shown in Figure 1.

\footnotetext{
${ }^{1}$ Corresponding Author: U.gunputh@derby.ac.uk
} 

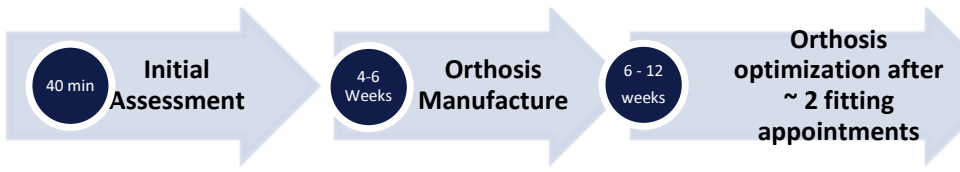

Figure 1: Timeline for NHS patients to receive orthosis

This paper describes a method embodied within a technology demonstrator to automate the design and fabrication of bespoke ankle foot orthoses, AFO, for patients within $48 \mathrm{hrs}$. Within this cycle time, the digital technologies adopted include 3D motion tracking to clinically assess the patient need using gait analysis, digital design modelling, fabrication by $3 \mathrm{D}$ printing and patient fitting. The digital methods and tools applied, demonstrate a technology with the potential to deliver affordable mass-customised healthcare devices which could transform patient healthcare. A smart factory is envisaged which could be a scanning booth where digital data are collected, analysed, transferred to a cloud database, the device 3D printed locally at a bureau and shipped to a surgery to fit to the patient within $24-48 \mathrm{hrs}$. The paper further identifies the opportunities and challenges arising from the demonstration. This proposed smart factory works towards enhancing a patient's pathway within the NHS hence aligning with the 2015 NHS report whereby a comprehensive integrated service is expected to have a significant health, quality of life and economic benefits [1].

\section{Technology Demonstrator}

This technology demonstrator was based on a custom fitted hinged AFO supplied to us by the NHS Derby and Burton Hospitals Trust as shown in Figure 2a. The latter AFO was created using the traditional manufacturing method (Foam and box; Thermoforming). It consists of 24 separate components which is time consuming to combine. The AFO design used in this study was a non-hinged AFO (Figure 2b) based on a single component hinged AFO designed by Williams et al [4] which was $3 \mathrm{~d}$ printed in a single print (Patent Number: GB2578635A).

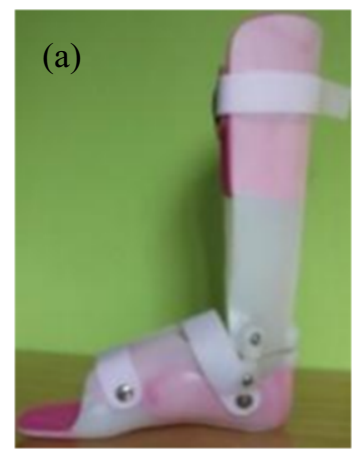

(b)

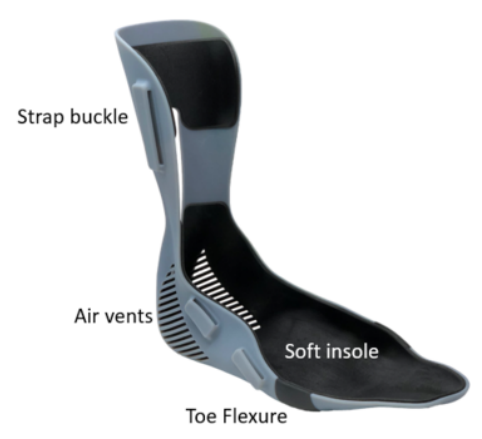

Figure 2: (a) Hinged AFO provided by the NHS and (b) new non-hinged AFO design based on Williams et al [4]

The demonstrator was based on the process outlined in Figure 1, from initial patient assessment to the final orthosis trial with a framework as Figure 3. 


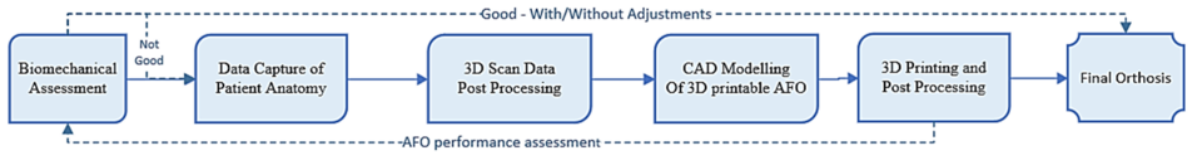

Figure 3: Proposed framework for demonstrator

\subsection{Biomechanical assessment}

In a clinical setting, once a patient's condition has been diagnosed by a health professional it is usually necessary to undertake some analysis of the patient's specific corrective needs to correctly specify the correct type and structural requirements for the AFO. For the purposes of our study a suitable able-bodied female adult with no significant requirement for correction was identified to assume the role of a patient. The biomechanical assessment of the patient's gait was completed using Visual3D software in conjunction with a dedicated 3D motion capture hardware set-up consisting of 9 infrared motion tracking cameras, multiple reflective targets mounted upon the patient's body at pre-determined repeatable locations (Figure 4a) and floor mounted pressure

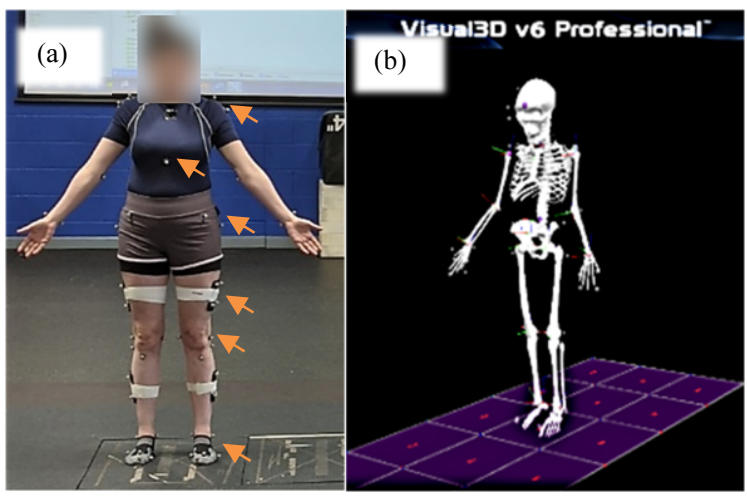

(c)

QNT CYCLE XNEPATKCS - RLGHT LEG
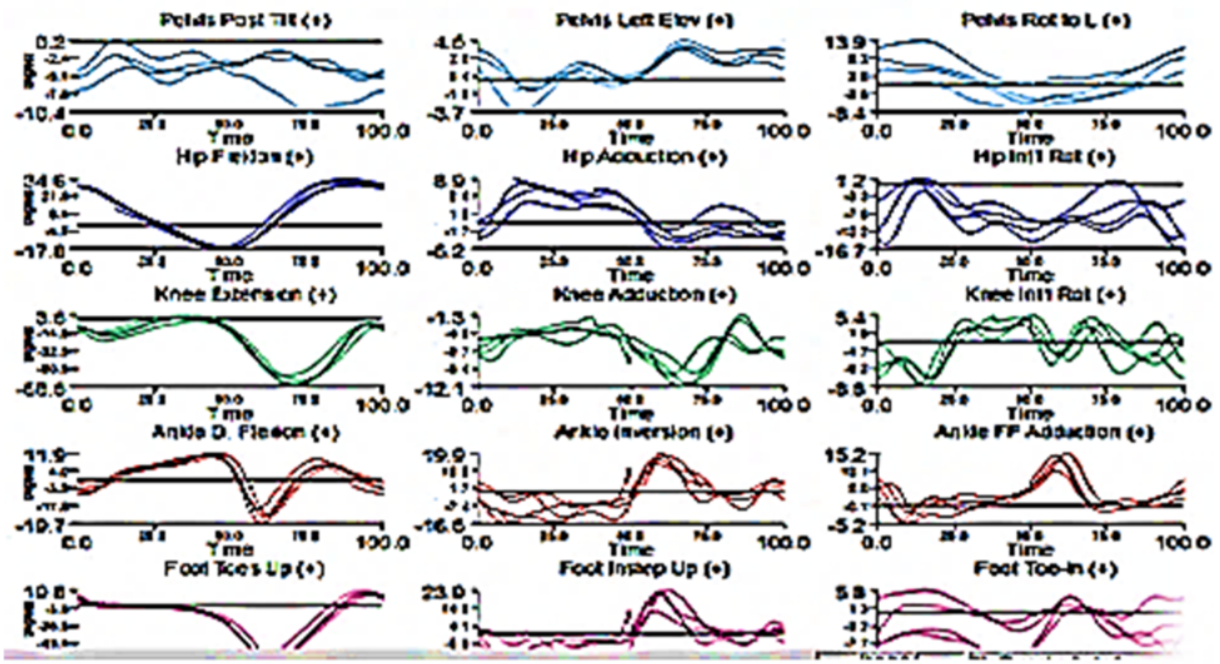

Figure 4: (a) Reflective targets on patient, (b) the corresponding biomechanical model as perceived by the Visual 3D software and (c) a sample metrics output from the software (Right Leg). 
sensitive plates. The patient walked over the pressure sensitive plates while sensors and cameras tracked movements (Figure 4b) and captured metrics in real-time (Stride length, Step width, Foot rotation, Gait speed and Stance ratio (Symmetry between the left and right-hand side of the body)). The process took one hour.

\subsection{Data capture of patient anatomy}

$3 \mathrm{D}$ scanning is known to be less prone to error and can be repeated more accurately as compared to box and plaster casting, regardless of clinical experience [5]. In this work, the patient's lower leg anatomy was captured using an Artec Leo portable handheld 3D laser scanning device ( 2 million points per second at a resolution of up to $0.5 \mathrm{~mm}$, working distance range is between $35 \mathrm{~mm}-1200 \mathrm{~mm}$ with $3 \mathrm{D}$ point accuracy of $0.1 \mathrm{~mm}$ ). The device is suited to this application as the speed of data capture ensured the patient was capable of comfortably maintaining the required foot/leg position for the entire duration of the scan, an additional benefit to using this system was the on-board highdefinition display affording the operator convenient real-time visualisation of the data capture which allowed constant review of scan progress. Accuracy of the resulting point cloud data met our target tolerance range for the anatomical data required for subsequent CAD modelling of the AFO. This step required $45 \mathrm{~min}$.

\section{3. $3 D$ scan data post-processing}

The output from the Artec Leo 3D laser scanner is point cloud data. A minimum of 3 scans were taken to capture maximum geometrical features. Using the Artec Leo 3D software, the scans were individually cleaned to remove noise, aligned and merged to create a watertight mesh file before being exported to PolyWorks Modeler (Innovmetri Software). The NURBS (Non-uniform rational B-spline) surface was created by mapping 3D scan which allowed the creation of the NURBS model. The whole process took $3 \mathrm{hrs}$.

\subsection{CAD Modelling of $3 D$ Printable AFO}

Outlines of the AFO shape were drawn on the NURBS model of the foot which were then further developed and trimmed accordingly (Figure 5). In this work, individual lines, contours, extrusion and cuts were done manually using available software, Solidworks, which needed $12 \mathrm{hrs} 30 \mathrm{~min}$.

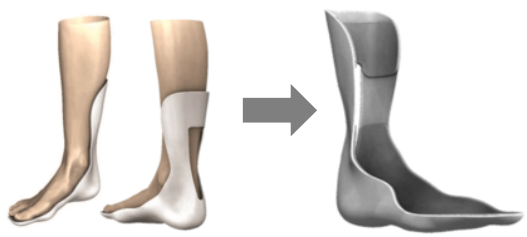

Figure 5: CAD Modelling of AFO

\section{5. $3 D$ Printing and Post-Process}

A multi-material 3D printer, Connex3 Objet500, was used for the purpose of this demonstration. The CAD model of the AFO, saved as an .stl file, was imported into the preparation software for the $3 \mathrm{D}$ printer, GrabCAD Print. The model was assigned a 
horizontal position on the build tray as shown in Figure 6 to provide optimum strength and minimise the use of support structures.

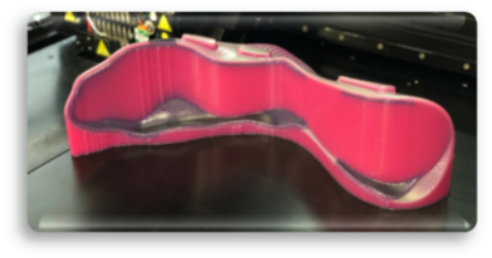

Figure 6: Location of $\mathrm{AFO}$ on build tray in 3D printer

The multi-material printer, with a build tray size of 500 by 400 by $200 \mathrm{~mm}$, uses Stratasys' Polyjet technology and can print more than one material (Thermoset polymer) in a single print. The layer-by-layer process involves deposition and curing (UV) of layers with a minimum thickness of 16 microns (30 microns for multi-material printing) with a build resolution of 600 dpi in the $\mathrm{X}$ and $\mathrm{Y}$ axis and 1600 dpi in the $\mathrm{Z}$ axis. The support material is a photopolymer namely FullCure 706 which can be easily removed. For the AFO, two materials were used namely Agilus 30 (Shore Hardness: 30-35 (A)) and Rigur (Shore Hardness: 80-84 (D)). Rigur being the more rigid material was selected for the main body of the orthosis while Agilus 30, being a softer material with more flexibility was used as an inner insole for comfort and to allow toe flexion at the bottom of the latter. The build time for one AFO was $45 \mathrm{hrs}$ with an additional $4 \mathrm{hrs} 30 \mathrm{~min}$ for post processing to remove support material which involved water jetting (Gemini Genie 460) and soaking the AFO in a caustic soda solution (CleanStation DT3).

Once the AFO was complete, a fit and biomechanical assessment was carried out with the patient to evaluate the performance of the orthosis, this task was completed in two hrs.

\section{Opportunities and Challenges}

The full duration for the manufacture of a single 3D printed AFO was $68 \mathrm{hrs} 45 \mathrm{~min}$. For mass production, this process can be optimised to be completed within $15 \mathrm{hrs}$ as depicted in Figure 7.

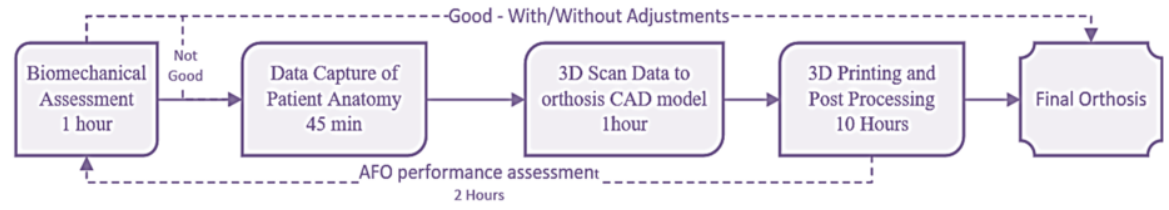

Figure 7: Framework for proposed cycle with timeline

The scanned data could be automatically converted efficiently using specialist software such as MediACE3D ${ }^{\mathbb{R}}$ within one hour. For the $3 \mathrm{D}$ printing process, if a larger printer is used with an increased build volume, more than one AFO could be built in a single print. Assuming 10 pieces are printed on one build plate, the overall length of printing will be greater but there will be a potential 35-hour reduction in the total print time per orthosis. A similar system could be installed within the orthotic department in every hospital targeting different types of orthoses, not limited to AFOs. One person could lead the whole process with minimal support in one location, reducing the manpower involved in the process. More patients could be treated within a few days with quick results hence 
reducing a patient's waiting time in the NHS [3]. Patients will be receiving their orthoses quicker which prevents secondary complications and enhance their quality of life. Paediatric patients will also have time to get used to their new orthoses before outgrowing them. Additionally, with the adoption of 3D printing, the orthosis could be personalised, for example with initials/names or images/logos embedded to the wearer's specification.

Nonetheless these increase the cost of the orthosis being manufactured. A Stratasys Connex3 Objet500 is a significant financial commitment, with expensive materials and running costs. For this demonstration, the print of a single AFO more than $70 \%$ of the total material used was support material which cannot be reused. For mass production, an alternative printer would be more economical, or possibly an alternative $3 \mathrm{D}$ printing process which is less reliant on support material such as selective laser sintering or multijet fusion. This will also lead to lower greenhouse gas emissions.

Any material being used needs to be mechanically tested in different conditions such as different UV exposure, humidity, temperature, and $\mathrm{pH}$. The mechanical test will have to simulate the function of the orthosis such as the BRUCE method used for AFOs as described in Bregman et al [6]. This process will help ensure the orthosis is fit for purpose and able to maintain the lifespan required by the patient.

\section{Conclusion}

A smart process including initial patient's assessment to patient receiving final working orthosis has been described in this paper. A demonstration successfully highlighted the significant reduction in a patient's waiting time thus potentially enhancing a patient's pathway when attending the orthotic department of the NHS. Various challenges have been identified with potential solutions explored.

\section{References}

1. Churchill, N. and C. Regan, Improving the Quality of Orthotic Services in England. 2015, NHS England: Online; england.nhs.uk/commissioning/wp-content/uploads/sites/12/2015/11/orthcsfinal-rep.pdf.

2. Nester, C.J., et al., National profile of foot orthotic provision in the United Kingdom, part 1: practitioners and scope of practice. Journal of Foot and Ankle Research, 2017. 10(1): p. 35 DOI: 10.1186/s13047-017-0215-4

3. Chockalingam, N., N. Eddison, and A. Healy, Cross-sectional survey of orthotic service provision in the UK: does where you live affect the service you receive? BMJ Open, 2019. 9(10): p. e028186 DOI: 10.1136/bmjopen-2018-028186

4. Williams, G., P. Wood, and J. Clementson, Joint orthosis with resiliently deformable hinge, I.P. Office, Editor. 2018: United Kingdom. p. 32.

5. Carroll, M., M.-E. Annabell, and K. Rome, Reliability of capturing foot parameters using digital scanning and the neutral suspension casting technique. Journal of foot and ankle research, 2011. 4(1): p. 9-9 DOI: 10.1186/1757-1146-4-9

6. Bregman, D., et al., A new method for evaluating ankle foot orthosis characteristics: BRUCE. Gait \& posture, 2009. 30: p. 144-9 DOI: 10.1016/j.gaitpost.2009.05.012 